\title{
TOTALLY UMBILICAL CR-SUBMANIFOLDS OF A KAEHLER MANIFOLD
}

\author{
By Sharief Deshmukh and S. I. Husain
}

\begin{abstract}
A classification Theorem for totally umbilical CR-submanifolds of a Kaehler manifold is proved.
\end{abstract}

\section{Introduction.}

$C R$-submanifolds of a Kaehler manifold [1] being generalization of holomorphic and totally real submanifolds of a Kaehler manifold, has recently become subject of sufficient interest. Totally umbilical $C R$-submanifolds of a Kaehler manifold have been studied by $A$. Bejancu [3], Blair and Chen [4]. The purpose of this paper is to classify all totally umbilical $C R$-submanifolds of a Kaehler manifold. In fact we prove the following theorem.

THEOREM. Let $M,(\operatorname{dim} M \geqq 5)$ be a complete simply connected totally umbilical $C R$-submanifold of a Kraheler manifold $\bar{M}$. Then $M$ is one of the following.

(i) Locally the Riemannian product of a holomorphic submanifold and a totally real submanifold of $\bar{M}$

(ii) totally real submanifold

(iii) isometric to an ordinary sphere

(iv) homothetıc to a Sasakian manifold.

The cases (iii) and (iv) occur when $\operatorname{dim} M$ is odd.

\section{Preliminaries.}

Let $\bar{M}$ be an $m$-dimensional Kaehler manifold with almost complex structure $J$. Then the curvature tensor $\bar{R}$ of $\bar{M}$ satisfies [11].

$$
\bar{R}(J X, J Y) Z=\bar{R}(X, Y) Z, \bar{R}(X, Y) J Z=J \bar{R}(X, Y) Z .
$$

An $n$-dimensional submanifold $M$ of $\bar{M}$ is said to be a $C R$-submanifold if on $M$ there exist two orthogonal complementary distributions $D$ and $D^{\perp}$ such that $J D=D$ and $J D^{\perp} \subset \nu$, where $\nu$ is the normal bundle of $M[1]$. If $D=\{0\}$, (resp.

Received April 14, 1986 
$D^{\perp}=\{0\}$ ), then $M$ is said to be totally real (resp. holomorphic) submanifold. It follows that $\operatorname{dim} D=$ even and that the normal bundle $\nu$ splits as $\nu=J D^{\perp} \oplus \mu$, where $\mu$ is invariant sub-bundle of $\nu$ under $J$. The Riemannian connection $\bar{\nabla}$ on $\bar{M}$ induces the connections $\nabla$ on $M$ and the normal connection $\nabla^{\perp}$ in $\nu$ obeying the Gauss and Weingarten farmulae

$$
\begin{aligned}
& \bar{\nabla}_{X} Y=\nabla_{X} Y+h(X, Y), \\
& \bar{\nabla}_{X} N=-A_{N} X+\nabla_{X}^{\perp} N,
\end{aligned}
$$

where $X, Y$ are vector fields on $M, N \in \nu$ and $h, A_{N}$ are called the second fundamental forms related as

$$
g(h(X, Y), N)=g\left(A_{N} X, Y\right) .
$$

The $C R$-submanifold $M$ is said to be totally umbilical if

$$
h(X, Y)=g(X, Y) H,
$$

where $H=\frac{1}{n}$ (trace $h$ ), called the mean curvature vector. For totally umbilical $C R$-submanifold $M$, the equations (2.2) and (2.3) take the form

$$
\begin{aligned}
& \bar{\nabla}_{X} Y=\nabla_{X} Y+g(X, Y) H \\
& \bar{\nabla}_{X} N=-g(H, N) X+\nabla_{X}^{\perp} N .
\end{aligned}
$$

The equation of Codazzi for totally umbilical $C R$-submanifold $M$ is given by

$$
\bar{R}(X, Y ; Z, N)=g(Y, Z) g\left(\nabla_{X}^{\frac{1}{X}} H, N\right)-g(X, Z) g\left(\nabla_{\bar{Y}}^{\frac{1}{H}} H, N\right),
$$

where $\bar{R}(X, Y ; Z, N)=g(\bar{R}(X, Y) Z, N)$ and $X, Y, Z$ are vector fields on $M$ and $N \in \nu$.

By an extrinsic sphere we mean a submanifold of an arbitrary Riemannian manifold which is totally umbilic and has nonzero parallel mean curvature vector [10]. We need the following Theorem of Yamaguchi, Nemoto and Kawabata [13].

"A complete connected and simply connected extrinsic sphere $M^{n}$ in a Kaehler manifold $\bar{M}^{2 m}$ is one of the following:

1. $M^{n}$ is isometric to an ordinary sphere

2. $M^{n}$ is homothetic to a Sasakian manifold

3. $M^{n}$ is totally real submanifold and the $f$-structure is not parallel in the normal bundle."

\section{Proof of the Theorem.}

Let $M$ be totally umbilical $C R$-submanifold of a Kaehler manifold $\bar{M}$. Then using (2.5), (2.6) and $J \bar{\nabla}_{X} W=\bar{\nabla}_{X} J W$ for $X, W \in D^{\perp}$, we get

$$
J \nabla_{X} W+g(X, W) J H=-g(J W, H) X+\nabla_{X}^{\perp} J W .
$$


Taking inner product with $X$ we get

$$
g(H, J W)\|X\|^{2}=g(X, W) g(H, J X) .
$$

Interchanging the role of $X$ and $W$ in above equation we get

$$
g(H, J X)\|W\|^{2}=g(X, W) g(H, J W) .
$$

Using (3.2) in above equation we have

$$
g(H, J W)=\frac{g(X, W)^{2}}{\|X\|^{2}\|W\|^{2}} g(H, J W) .
$$

The possible solutions of equation (3.3) are:
(a) $H=0$ or
(b) $H \perp J W$, or
(c) $X \| W$.

Thus we have one of the following:

(a) $M$ is totally geodesic, (b) $H \in \mu$ (c) $\operatorname{dim} D^{\perp}=1$.

Combining (a) with a result in [4] we get part (i) of the Theorem.

Next suppose that $H \neq 0$ and $H \in \mu$. We observe that for $N \in J D^{\perp}$ and $X \in D$, $\bar{\nabla}_{X} J N=J \bar{\nabla}_{X} N$ gives $\nabla_{X} J N=J \nabla_{X}^{\perp} N$. This implies that for $N \in J D^{\perp}$ and $X \in D$, $\nabla_{X}^{\perp} N \in J D^{\perp}$. Also $g(N, H)=0$ for $N \in J D^{\perp}$ implies $g\left(\nabla_{X}^{\perp} N, H\right)=-g\left(N, \nabla_{X}^{\perp} H\right)$, this together with $\nabla_{X}^{\perp} N \in J D^{\perp}$ gives $g\left(N, \nabla_{X}^{\perp} H\right)=o$. Hence for $X \in D$, we get $\nabla_{X}^{\perp} H \in$ $\mu$. Now for $X \in D$, we have from $\bar{\nabla}_{X} J H=J \bar{\nabla}_{X} H$, with the help of (2.6), that

$$
\nabla_{X}^{\frac{1}{X}} J H=-g(H, H) J X+J \nabla_{X}^{1} H .
$$

Since $\nabla_{X}^{1} H \in \mu$, from (3.4) it follows that $J X=0$ for all $X \in D$. Hence $D=\{0\}$, this proves part (ii) of the theorem.

Lastly suppose $H \neq 0, H \oplus \mu$ and that $\operatorname{dim} D^{\perp}=1$. Since $\operatorname{dim} M \geqq 5$, we can choose vectors $X, Y \in D$ such that $g(X, Y)=g(X, J Y)=0$. Now from (2.7) it follows that $\bar{R}(J X, Y ; J Y ; N)=0, N \in \nu$. Using (2.1) we get $\bar{R}(J Y, X ; J Y, N)$ $=0$. This, with the help of (2.6) gives

$$
g\left(\nabla_{X}^{\perp} H, N\right)=0 \forall N \in \nu .
$$

This proves that $\nabla_{X}^{\perp} H=0$ for $X \in D$. Next we let $X \in D^{\perp}$. Then there exists a normal $N^{\prime}$ such that $J X=N^{\prime}$. Now for $N \in \mu$ we have $\bar{R}(X, Y ; J Y, J N)=0$, $Y \in D$. Using (2.1) in this we get $\vec{R}(X, Y ; Y, N)=0$ and this together with (2.7) gives $g\left(\nabla_{X}^{\perp} H, N\right)=0$, from which it follows that $\nabla_{X}^{\perp} H \in J D^{\perp}$. Now again from (2.7) and (2.1) we have $\bar{R}(X, Y ; Y, X)=\bar{R}\left(X, Y ; J Y, N^{\prime}\right)=0, N^{\prime}=J X \in J D^{\perp}$. Using linearity of $\bar{R}$ in $\bar{R}(X, Y ; Y, X)=0$, we get $\bar{R}(X, Y ; J Y, X)=0$. This gives $\bar{R}\left(X, Y ; Y, N^{\prime}\right)=0$. From this using (2.7) we get $g\left(\nabla_{X}^{\frac{1}{X}} H, N^{\prime}\right)=0$. From this it follows that $\nabla_{X}^{\perp} H \in \mu$. Thus we have proved for $X \in D^{\perp}, \nabla_{X}^{\perp} H \in J D^{\perp} \cap \mu$ $=\{0\}$, i.e. $\nabla_{X}^{\perp} H=0$. Hence $\nabla_{X}^{\perp} H=0$ for all vector fields $X$ on $M$ i.e. $M$ is an extrinsic sphere. Then parts (iii) and (iv) of the Theorem follow from theorem 
of Yamaguchi, Nemoto and Kawabata in $\S 2$.

This theorem thus gives a complete classification of totally umbilical $C R$ submanifolds of a Kaehler manifold.

\section{Remark.}

In case of complex space form $\bar{M}(c)$ i.e. Kaehler manifold of constant holomorphic sectional curvature $c$, the curvature tensor $\bar{R}$ of $\bar{M}(c)$ is given by

$$
\begin{aligned}
\vec{R}(X, Y) Z= & \frac{c}{4} g(Y, Z) X-g(X, Z) Y+g(J Y, Z) J X \\
& -g(J X, Z) J Y+2 g(X, J Y) J Z .
\end{aligned}
$$

If $M$ is totally umbilical submanifold of $\bar{M}(c)$ and $R$ is curvature tensor of $M$, then by Gauss equation we have

$$
g(R(X, Y) Z, W)=g(\bar{R}(X, Y) Z, W)+\alpha[g(Y, Z) g(X, W)-g(X, Z) g(Y, W)]
$$

where $\alpha=g(H, H)$.

By [10] or [2] a totally umbilical submanifold of $\bar{M}(c)$ is either holomorphic submanifold or a totally real. Thus we have a corollary in light of equation (4.2).

COROLlary. Let $M$ be totally umbilical submanifold of a complex space form $\bar{M}(c)$. Then $M$ is one of the following

(i) a complex space form $M(c)$

(ii) a totally real submanifold of constant curvature $c$

(iii) a totally real submanifold of constant curvature $c+\alpha$.

This corollary is essentially theorem due to Chen and Ogiue [10].

\section{REFERENCES}

[1] A. Bejancu, CR-submanifolds of a Kaehler manifold, Proc. Amer. Math. Soc., 69 (1978), 135-142.

[2] A. Bejancu, CR-submanifolds of a Kaehler manifold-II, Trans. Amer. Math. Soc., 250 (1979), 333-345.

[3] A. Benjancu, Umbilical CR-submanifolds of a Kaehler manifold, Rend. Mat., 13 (1980), 431-466.

[4] D.E. Blair and B.Y. Chen, On CR-submanifolds of Hermitian manifolds, Israel J. Math., 34 (1980), 353-363.

[5] B. Y. Chen, Geometry of submanifolds, Dekker, N.Y. (1973).

[6] B. Y. CHEN, Extrinsic spheres in Kaehler manifolds, Mich. Math. J., 23 (1976), 327-330.

[7] B.Y. Chen, Geometry of submanifolds and its applications, Sci. Univ. Tokyo, Tokyo (1981). 
[8] B. Y. Chen, On CR-submanifolds of Kaehler manifolds, I, II, J. Diff. Geom., 16 (1981), 305-322, 16 (1981), 493-509.

[9] B. Y. Chen And K. Ogiue, On totally real submanifolds, Trans. of Amer. Math. Soc., 193 (1974), 257-266.

[10] B. Y. Chen and K. Ogiue, Two Theorems on Kaehler manifolds, Michigan Math. J., 21 (1974), 225-229.

[11] M. OватA, Riemannian manifolds admitting a solution of a certain system of differential equations, Proc. U.S. Japan Seminar in Differential Geometry (Kyoto, 1965), 101-114.

[12] M. OKumura, Certain almost contact hypersurfaces in Kaehler manifold of constant holomorphic sectional curvature, Tohoku Math. J., 16 (1964), 270-284.

[13] S. Yamaguchi, H. Nemoto and N. Kawabata, Extrinsic spheres in a Kaehler manifold, Michigan Math. J., 31 (1984), 15-19.

[14] K. Yano, Geometry of complex and almost complex spaces, Pergaman Press, N. Y. 1965.

Department of Mathematics

Aligarh Muslim University

AligarH-202001 (INDIA) 